mater did not appear to be diseased. The antrotympanic cavity was full of cholesteatoma. At first the patient made sutisfactory progress. On the eighth day after the operation she complained of headache and vomited soon after her dinner, then was quite bright and comfortable. On the ninth day she again complained of headache and in the afternoon looked ill. Her pulse was found to be 60 per minute about 4 o'clock, at 5 o'clock it was only 50 per minute, and the patient was growing drowsy. About 8 P.M. the patient was very drowsy, the knee-jerks were increased, ankle clonus was present, the pulse was 50 , and the right pupil was dilated and fixed, while the left was normal; there was no optic neuritis. The mastoid wound was then re-opened, more bone removed from the floor of the middle fossa, and a large abscess was found in the temporo-sphenoidal lobe. On opening the abscess a considerable quantity (unfortunately not measured) of stinking greenish fluid gushed out. A large drainage-tube was introduced. The right pupil immediately came down to normal size and next morning ankle clonus, \&c., had disappeared. Two days later left hemiplegia, beginning at the face and extending to the foot, developed during the night. In order to relieve intracranial pressure bone was removed from the side of the skull over the temporo-sphenoidal region. This had no beneficial effect on the hemiplegia. The brain began to herniate through both this wound and that on the floor of the middle fossa and continued to do so for several days. The patient gradually sank and died on the twelfth day after the opening of the cerebral abscess.

CASE 3. - The patient, aged 24 years, was carried into the Brighton Throat and Ear Hospital in a comatose condition on March 18th, 1906. Practically no history could be obtained except that he had suffered from otorrhœa for a long time, that he had complained of earache and beadache for three or four days, that he looked ill on March 17th, and was beginning to grow drowsy on the morning of the 18th. When brought to hospital he put his tongue out when ordered in a loud voice to do so, and answered "All right now" to questions. The head-was slightly retracted and the neck stiff. Both ears were suppurating; in the right membrana tympani there was a large clean-cut perforation without any granulation tissue, while the fundus of the left ear was blocked with granulation tissue. A careful examination of the eyes, of the reflexes, \&c., gave no information as to the probable site of the intracranial mischief; but as the fundus of the left ear was blocked with granulations, whereas the right ear was free, it was decided to open the left mastoid. The cortex was very dense. On opening the left mastoid antrum a drop of pus was found oozing slowly through the tegmen. The tegmen was therefore removed freely, and thus a large extradural abscess was opened. A large drainage-tube was introduced and the wound dressed. On removing the dressings next day pus was found between the dura mater and the squamous portion of the temporal bone. A disc of bone was accordingly removed in order to ensure free drainage. The patient made a slow but satisfactory recovery. When he was last seen (several months after leaving the hospital) the operation wound was quite healed, the ear dry, and the patient's health good.

CASE 4.-The patient, aged 48 years, had an attack of acute left middle-ear inflammation which came on during a bad cold in the head in the last week of November, 1909. Severe pain in the ear lasted a night, but was much relieved when the membrane ruptured and discharge commenced. As the pain, though less severe, still continued, the patient applied several very hot poultices over the ear and mastoid region, making the skin very red over these parts and even raising some blisters, thus blurring the clinical picture. On Dec. 3rd the following conditions were found-viz. tenderness and slight swelling over the mastoid process; redness and slight bulging of the membrana tympani, in the postero-superior segment of which there was a small perforation; very little discharge; temperature $103.4^{\circ} \mathrm{F}$. The patient was removed to a nursing home and a "Schwartze" operation performed. The bone of the mastoid process was soft and red, and contained here and there little pockets of pus, and pus was found in the antrum. The patient made fairly satisfactory progress for a week, but on the seventh day after operation she complained of pain in the left temple and to a less degree over the whole of the left side of the head, and of a tender swelling behind the angle of the jaw on the same side, and the temperature rose to $102^{\circ}$. By the evening the temperature had fallen to $99^{\circ}$, but the patient remained in a restless, irritable condition. On the tenth day the temperature rose to $104^{\circ}$ and two rigors occurred. Examination of the heart, lungs, eyes, nerve reflexes, \&c., revealed nothing abnormal. On the same evening the mastoid wound was reopened and the bone removed back to the lateral sinus. This was found to be full of thrombus which was just beginning to break down (a pure culture of streptococcus pyogenes was grown from this, and a vaccine prepared, of which four injections were subsequently given to the patient). The internal jugular vein was divided between ligatures, the lateral sinus incised, and the clot turned out. Free hæmorrhage occurred from the occipital end of the sinus; this was controlled by plugging. Normal saline solution was then syringed up the jugular vein, whereupon hæmorrhage began at the anterior end of the opened sinus. This was plugged and the ligature round the vein tied. The whole wound was then painted with tincture of iodine-a proceeding which was repeated nearly every day till healing was practically complete. The patient made an uneventful recovery, and is now in good health.

Brighton.

\section{THE SPECIFICITY OF CANCER AND THE GENERAL PRINCIPLES OF ITS TREATMENT AND PROPHYLAXIS. ${ }^{1}$}

BY ALEXANDER THEODORE BRAND, M.D., C.M. ABERD., V.D.,

EX-PRESIDENT, EAST YORKS AND FORTH IINCOLN BRANCH, BRITISH MEDICAL ASSOCIATION; FELLOW OF THE ROYAI SOCIETY OF MEDICINE.

THE treatment of disease is at all times beset with difficulty even when the cause is well known, and this difficulty is greatly enhanced in the case of maladies whose cause is still undetermined. In the latter class treatment can be only empiric. The cause of cancer has not, so far, been proved to demonstration, and unfortunately no remedy of any kind, even empiric, has been discovered; but though the cause has not yet been demonstrated by the production of the originating agent (and such is not remarkable since we are merely on the threshold of the knowledge of the causation. of specific disease), still it is logically deducible from the well-known facts relating to the disease.

No disease more plainly bears upon itself the impress of specificity than cancer; indeed, this is one of its chief characteristics. Its constant primary local origin in practically any situation; its invariable extension by auto-infection, evidenced by its widespread dissemination by metastasis ; its constant relation to a " condition precedent" $;$ " the innumerable socalled " coincidences" which clearly point to what would, in any other diseased condition, be considered infection; and the countless successful inoculations which have been made, all testify to its true specific nature-i.e., to its being as typically infective a process as syphilis or tuberculosis.

Although analogy is quite incapable of proving anything, being merely a "resemblance in relations," yet even analogy is not wanting, for cancer is strikingly analogous to such parasitic diseases as syphilis, tuberculosis, and pyæmia, in spite of the fact that it possesses one peculiar idiosyncrasy of its own, inasmuch as its metastases are formed by proliferation of the transported cells of the primary focus. In all other similar diseases the infective agent is transported free, while in cancer it is conveyed encapsuled in a tissue cell. This is only another example of the extraordinary variety of parasites and their ways.

Strange to say, some non-believers in its exogenesis openly assert that cancer is a parasitic disease, but they allege that it is the cancer cell itself which is the parasite. They do not appear to be able to appreciate the obvious logical sequitur that, since no somatic cell can, per se. be a pathogenic parasite, there must of necessity be an intracellular influence

The "condition precedent" is a term introduced to indicate that state of vulnerability which must exist before an indiridual can be successfully attacked by the cancer parasite. It is induced $l, y$ degeneration of tissue from any cause, more especially from obsolescence of organs, senescence, and senility, chronic disease, continued irritation, traumatism, and by congenital susceptibility.-A. T. B. 
which endues it with its malign parasitic power. In other words, there must be an intracellular parasite, and all parasites are extrinsic.

Why the extrinsic origin of cancer is not universally accepted is to me incomprehensible in the face of evident facts; but the sooner it becomes generally received the sooner is the search for the causative agent likely to be crowned with success, and the stigma of its unsuccessful treatment to be removed from the practice of medicine. The concentration of research, based upon the logical hypothesis of exogenesis, must be more likely to be successful than the misdirected efforts of those who spend time and cerebral energy, not to speak of the great waste of money, in endeavouring to prove that a specific disease can possibly have an intrinsic origin, efforts which amount simply to beating the air.

It is generally admitted that all specific diseases, except ancer, arise by exogenesis-i.e., by infection from withoutand that there is a known or suspected infective agent or parasite in each case which, so far as our present knowledge goes, is a micro-organism, either a microphyte or a protozoon, and that these diseases are communicated directly from individual to individual, or indirectly by inanimate objects, or through an intermediate host. With regard to cancer, I am convinced of its origin also by exogenesis, and that the infective agent resides in the invaded cells, although it has hitherto escaped detection.

I do not propose here to discuss the interesting problem why the hypnthetical intracellular parasite of cancer has not been discovered, but I would remind you that the Treponema palludum, responsible for syphilis, has only very recently been isolated, and that the parasite of small-pox and its bovine modification vaccinia (as well as that of many other specific diseases) still remains perdu.

That the infective agent of cancer resides in the invaded cell appears to me to be indisputable, since a single cell from a cancerous tumour is capable of reproducing the disease in another host, as has been proved by very many successful experiments, and an infected cell in the same host is capable of establishing a fresh focus of disease in another situation, as is evidenced by the formation of metastases, and by the reappearance of the disease in, or near, a surgical cicatrix from cells left behind, or introduced into the wound by the surgeon's knife, which has set them free from the originating growth.

Further evidence of the existence of an intracellular malignant agent, or parasite, is derived from the fact that the parencbyma cells of a carcinoma have been observed by Ehrlich and Apolant to impart a malignant stimulus to the surrounding connective tissue cells, so giving rise to a true sarcoma. From this evidence it may also be deduced that one and the same species of parasite is sufficient to originate any and every variety of malignant neoplasm, such variety being determined by the nature of the tissue attacked, and limited only by the anatomical diversity of the tissues themselves. Hence the urgent necessity which exists for the detection of the elusive pathogenic principle, both in the invaded cells and in the lymph and blood.

The exact date of attack in cancer cannot be determined, for, unfortunately, there is no known initial or prodromal symptomatology, and the interval between invasion and extension by auto-infective dissemination is consequently unknown, but it is believed to vary with the situation and tissue of the primary focus and that it may be very short. It is obvious that if the date of infection could be known at the time, and if the site were accessible, immediate excision or other destruction of the invaded part would be tibe ideal method of treatment, for such might be confidently expected to be absolutely successful, just as syphilis can be successfully averted by the immediate destruction of its causative parasite at the site of inoculation. Such data, however, with regard to cancer, in our present state of knowledge, are available only in the case of experimental or deliberate inoculation.

The bar to success in treatment is that it is never called for until the disease has given unequivocal evidence of its existence as a distinct tumour, and even where appearances might suggest an early and purely local invasion, widely disseminated extension, hopelessly beyond the surgeon's reach, has in all probability already occurred. It should, therefore, be a surgical axiom that if any inaccessible focie.g., infected lymphatic glands-are known or suspected to exist, as metastases of an accessible primary neoplasm, operation with a view to the removal of the latter is not only inadvisable but unjustifiable, for such a procedure would be utterly futile for the eradication of the disease. Not only, indeed, would operation in such circumstances be futile and unscientific, but it would probably be the actual cause of increase in the activity of the malignant process, by setting free, and scattering broadcast, infected cells hitherto confined, so that the last state of the patient would be worse than the first, and even life itself would be materially shortened. The presence of inaccessible foci does not, however, negative purely palliative operative procedures, such as the relief of cedema of the arm by lymphangioplasty, as devised by Sampson Handley, or the removal of necrosed and offensive tissues.

Unless distant extension is obvious free excision of the accessible diseased area, including infected lymphatic vessels and glands and adjacent structures-e.g., muscles, and especially fascia-may be attempted as the first line of treatment (vis a fronte), the surgeon bearing in mind that every single infected cell left behind or set free by his knife is a potential agent in causing a local and general recrudescence of the disease.

Although very exceptional cases have been recorded where tumours, undoubtedly malignant, have disappeared spontaneously, and although surgical removal of such tumours has apparently cured the disease in some cases, it is more than doubtful if any operative treatment of accessible malignant neoplasms is ever successful in the complete eradication of cancer; for by the time that a tumour has been discovered far-reaching infection has almost certainly already taken place. But for the incidence of fatal intercurrent disease, the malignant process must have inevitably reasserted itself sooner or later and eventually claimed its victim.

It is a marked characteristic of cancer, and one which it shares with syphilis, that it may remain quiescent for a time. Thus, a distant metastatic focus may remain dormant for an indefinite period, the victim being lulled into a false sense of security, to light up at last into fatal activity, while the final site of the disease may be sc remote from the original focus as to mislead the surgeon as to its true nature.

As a concrete example of this I may quote a case of Sir Hector Cameron's. In 1894 he removed the left mamma from the patient of a medical friend. In 1903 this patient began to complain of so-called "rheumatic" pain in her left hip and thigh, which became so severe as to cause her to go to bed and have medical attendance. One day, on getting out of bed, the left femur spontaneously fractured. Her medical attendant could find no trace of cancer in any other part of her body, but the patient died from gradual exhaustion without any evidence of farther dissemination in lungs, liver, or bone. Here some infective particle, lymph conveyed, had metastatically resided in the medulla of the femur for nine years, making no sign, until at last waking into activity by quick growth gave rise to pressure, pain, destruction of hone, spontaneous fracture, and death from exhaustion. There can be no reasonable doubt but that if this patient had died during the interval from intercurrent disease-e.g., pneumonia - she would have been declared cured by operation of her original cancerous disease. Many other instances might be adduced where recurrence, subsequent to operation on the breast for malignant disease, has occurred in other situations e.g., the vertebræ, brain, \&c.- -and after even longer periods of quiescence, but that just quoted will serve as an example.

This period of dormancy suggests that all infected cells are not equally active-i.e., that the hypothetical intracellular parasite is not always in the same state of development, some cells containing it in an intermediate form, which form remains quiescent until some unknown stimulus or time and circumstances favour its further evolution. Snch a hypothesis would explain many otherwise inexplicable clinical facts relative to cancer.

It is evident, therefore, that want of success after surgical treatment must be attributed either to incomplete removal or to the occurrence of distant dissemination prior to operation

Unsatisfactory as surgical treatment is in cases of accessible primary disease, there can be nn hope from the surgeon where the neoplasm is inaccessible from the first or where it has shown itself to have become so after operation. 
Dark indeed must the prospect appear to the man who believes cancer to be a constitutional disease with local manifestations and having an intrinsic origin, for even the surgeon can do nothing for its removal. After all, the surgeon can only be expected to deal with what he can see, and temporarily stave off impending death threatened by mechanical causes; he cannot reach the cause. Cancer is practically ineradicable by surgical means alone, for the reason already stated. If cancer is ever to be successfully reated, the cause itself must be attacked, and this can be done only by specific means. This constitutes the second line of treatment (vis a tergo).

The surgeon confronted with a fully developed Hunterian ulcer is powerless to avert constitutional dissemination by the knife--indeed, it has already occurred. In like manner he cannot expect to eradicate cancer which has already become constitutional by local excision of its visible manifestation. The physician, on the other hand, in the case of syphilis, can view the local indication of the disease with equanimity, for he knows that he is able to combat constitutional disease successfully by proved specific remedies.

Cancer being as truly specific a disease as syphilis, and since the latter yields to internal specific treatment, it is reasonable to assume that the former will likewise be amenable to the same. In such treatment appears to lie the only hope of the victim of cancer, and well will it be for him when it has been established that this disease is due to an extrinsic cause, for recovery may then be hopefully looked for from appropriate remedies. Should the infective agent prove to be a protozoon, as is the case in syphilis, malaria, \&c., and $m$ belief is that the probability is in favour of such a parasite, then a parasiticide is indicated, similar in lethal action to those found effectual in these diseases. Among the methods of exhibiting such parasiticide kataphoresis of accessible neoplasms would doubtless commend itself.

The parasiticide specific for cancer must be sought for by experiment both on man and the lower animals; and I should imagine that investigators would find no more willing, nay zealous, coöperators than the human subjects of cancer themselves.

On the other hand, should the causal agent bo found to be a bacterium, success may follow the exhibition of a cancer vaccine, prepared after the methods of Wright; or of an anti-cancerous serum. Until such serum can be procured from an individual cured of, or rendered immune to, the disease, the effect of auto-serum might be tried. This may contain a self-generated antitoxin; or, if it contains the toxin itself, its injection in small amount may act as a vaccine and stimulate the leucocytes to increased phagocytic activity.

From the foregoing considerations I would submit that the successful treatment of cancer $w i l l$ consist in the removal by surgical means of accessible tumours, more especially those threatening life from mechanical causes, combined with the internal admitistration of specific remedies, the latter being relied upon to check the furth $\mathrm{r}$ spread of disease and neutralise that already existing, by destroying the causal parasite; while inoperable disease, whether accessible or inaccessible from the first, can only be treated by specific remedies and such non-operative mechanical means as fulguration, $X$ rays, radium emanations, kataphoresis, \&c.

No less important, or even more important, than the curative treatment of cancer is its prophylaxis. It is a truism that " prevention is better than cure," although this naturally is not the dominant sentiment of the sufferer from the disease.

Everything that tends to the encouragement of the "condition precedent" should be avoided. We cannot, indeed, stay the flight of time, that edax rerum which brings in its wake senescence, senility, and the obsolescence of organs, which play so important a part in the incidence of cancer, but these may be greatly mitigated by a strict observance of all the well-known laws of health.

Cremation of the cancerous cadaver is imperative, since such would remove a very obvious risk of perpetuation of the cause of the disease when earth burial is resorted toe.g., by the action of earthworms, which convey soil frem a depth of 6 to 8 feet to the surface, and by the contamination, by percolation, of the sources of water-supply. All dressings of cancerous wounds, all discharges and dejecta from cancerous patients should be destroyed, preferably by fire. There should be thorough disinfection of all rooms and houses inhabited by those suffering from, or who have died from, cancer. Intimate personal contact with the diseased should be avoided as far as possible. Personal cleanliness should be scrupulously observed, especially in the washing of the hands before eating, and in the preparation of food. There should be perfect sterilisation of all water and food partaken of, especially when the latter is eaten in an uncooked condition; and the contamination of any food by the lower animals, or by flies, should be prevented.

If there is any truth in the allegation that the earthworm is an intermediate host, and that the soil is one habitat (water being another) of the parasite, the mere idea of eating raw vegetables, however well scrubbed and apparently clean, becomes repulsive, and is calculated to induce one to ponder over the desirability or otherwise of total abstinence from all uncooked fruits and vegetables which lie on or in the surface of the ground.

Mr. J. Bland-Sutton, in his recent work "Cancer Clinically Considered," says :-

The frequency with which cancer attacks the large intestine is in favour of those who believe that cancer is due to a micro-parasite, probably something which is taken in with uncooked food or with water. Indeed. I feel so strongly on tbis matter that for many years I have avoided eating all sorts of uncooked vegetables. Much as I enjoy salad with my chicken, or my cheese, I do not touch it. However clean your celery may appear to be, if you pull aside the stalks immediately round the centre, which is about the only part worth eating, you will see that there remains some dirt which has not been washed away. One feels deeply thankful that most of the food eaten by mankind is cooked. Partially cooked or "underdone" meat carries penalties in the way of entozoa, and Metchnikoff says that "entozoa may serve as gates of entry for the hypothetical parasites of carcinoma." Food and water are essential to life, and it is undeniable that they are the frequent purveyors of disease, and that minute animal parasites are our dailv messmates and table companions, though their presence is sublimely ignored by us. In Behla's opinion the probable vehicle of infection in cases of cancer occurring in Luckau in Germany was the uncooked vegetables.

Finally, notification of all cases of cancer should be enfurced.

Driffield.

\section{ON THE DEMONSTRATION AND STUDY OF SPORES IN THE SCHIZOMYCETES.}

By A. F. STANLEY KENT,

PROFESSOR OF PHYSIOLOGY IN THE UNIYERSITY OF BRISTOL.

THE demonstration of spores within the cell body of the schizomycetes has been attempted in different ways by different workers. At the present time, however, and even by modern methods, the process is tedious and uncertain, and, as is often the case with methods wbich do not depend upon accurately known chemical and physical processes, the preparation of satisfactory specimens calls for an excessive expenditure of time and care. Moreover, some of the methods which have been proposed result in a considerable amount of damage being done to the rest of the cella damage which may seriously impair the usefulness of the preparations, whilst investigators working upon spore formation find the prolonged methods necessary a great drawback.

Bearing these facts in mind, and happening to be working on the subject some few years ago, I considered the various processes used and the explanations given of their supposed sole, when it occurred to me that the old idea of there being an envelope, or film, of a fatty nature in the neighbourhood of the spore, shutting it off from evaporation and from contact with aqueous solutions, whilst providing some explanation of the difficulty of decolnurising already stained spores, might also give some indication of a method for enabling the stain to penetrate more easily. Iy experiments were often interrupted, but the indicati ns given above being followed, a method was ultimately + laborated which enabled the more easily stainable spores to be coloured readily, whilst in the case of those which usually are coloured only with difficulty, the process is rendered easier.

The method at first consisted in the snlution of the staining agent in a fatty stlvent, and some success was obtained in this way, but soon another procedure was a topted and a different principle empliyed. The peculiarity of the method consisted in the employment of an alkali for 